\title{
Advanced image analysis of stem cells and tumor initiating cells
}

\section{Keywords: stem cells, digital pathology, oncology; TICs, CNT}

\section{Introduction}

Digital pathology and image analysis studies are growing rapidly in prevalence and sophistication, due to advances in multiplexed immunohistochemical techniques, whole slide imaging of tissue, and dealing with the complexities of personalized medicine. Oncology in particular has seen large investment in image analysis as a way to identify those patients likely to respond for a given therapy, especially in immune oncology programs where the parameters for an optimum patient selection paradigm may be impossible to measure using traditional pathology approaches. ${ }^{1}$ In this article, we review the state of image applications to another growing scientific field, the study of stem cells and their malignant analogs, so-called "cancer stem cells" or more appropriately "tumor initiating cells" (TICs). As TICs are thought to be in many cases the main drivers of tumor recurrence, therapeutic resistance, and metastasis, accurate quantitative assessment of their existence along with related biomarker expression in a tissue context will be essential to the design of therapeutic approaches that can deliver significant improvements in long term patient survival. ${ }^{2-4}$

Telomapping combined with advanced spatial recognition approaches allows the identification of adult stem cells within their native niches (unpublished results), which is of great relevance for regenerative medicine and oncology in general. Telomerase activity is a critical and unique aspect of stem cell function, and essential to experimental induction of stem cell characteristics in induced pluripotent stem cells (iPSCs). Telomere length is the most straightforward readout of telomerase activity, and can be measured accurately by image analysis of slides prepared using such a telomapping approach. Studies highlighting the significant lifespan increase in mice through telomerase gene therapy ${ }^{5}$ or the rejuvenating effects of telomere elongation ${ }^{6}$ have used this approach, which is based on Definiens' Cognition Network Technology (CNT) image analysis methods. ${ }^{7}$ In multiplexed IF images of histological sections of organs, nuclei are segmented based on their DAPI signals. Based on their spatial patterns, hierarchical super-structures - such as villi in mouse intestine and Lieberkühn crypts at their bottom - are identified, which allow the specific topological assessment of their nuclear subobjects. Within every nucleus, individual telomere sub-structures are segmented and telomere length is quantified as a function of the signal intensity of a fluorescently labeled PNA-telomeric probe. The method facilitates not only a binary determination of the stemness of cells in histological sections, but allows a detailed, continuous quantification of telomere length. Cells with longest telomeres characterize most primitive adult stem cells, while shorter telomeres occur usually mark the more differentiated compartments in a given tissue. ${ }^{8}$ The detection and characterization of stem cells in healthy or disease conditions can contribute to a better understanding of treatment response.

Quantitative and spatial assessment of TICs has particular relevance to personalized medicine, both in terms of targeting of the TICs themselves and immunotherapies. TICs, like normal adult stem
Volume 2 Issue 6 - 2017

\author{
Brian Laffin,' Johannes Zimmermann² \\ 'Associate Director, Translational Diagnostics, USA \\ ${ }^{2}$ Director Tissue Phenomics Datafication, Definiens AG, Munich, \\ Germany
}

Correspondence: Brian Laffin, Associate Director, Translational Diagnostics, Definiens Inc., 125 Cambridge Park Drive Ste. 300, Cambridge, MA 02I 40, USA, Tel +I (78I) 859 8896, Email blaffin@definiens.com

Received: June 0I, 2017 | Published: June 14, 2017

cells, have immune privileged status and therefore may be a source of resistance to immunotherapy in addition to their other tumor-promoting properties. ${ }^{3,4,9,10}$ CNT-based image analysis was used to trace down in clinical samples a specific subpopulation of putative breast cancer TICs using duplex IHC. ${ }^{11}$ This study demonstrated that the prevalence of $\mathrm{CD} 44^{+} / \mathrm{CD} 24^{-/ \text {low }}$ cells, which are in their capabilities to self-renew and proliferate similar to normal stem cells, ${ }^{12}$ is not directly linked to tumor progression, but might be associated with distant metastasis and other facets of tumor biology in multiple solid tumor types. ${ }^{13,14}$ CNT has also been employed to investigate the TIC composition of HGF-driven lung tumor models, and forge a mechanistic link between HGF/MET induced EMT (epithelial-mesenchymal-transition) and the TIC populations observed. ${ }^{15}$ Image analysis in this context can help go beneath the surface of tissue-based experiments, providing quantitative information on TICs that could help inform treatment decisions in a tumor-specific manner. Once target populations are identified, further image analysis studies of TICs can provide quantitative target engagement data, as shown in the case of FAK inhibition in breast cancer TICs. ${ }^{2}$ Additionally, CNT helped improve the definition of TICs in renal cell carcinomas, where automated assessments of tumor cells demonstrated underestimation of TICs in $\mathrm{RCC}$ and highlighted shortcomings in limited dilution assays frequent used for the experiment identification of TIC populations. ${ }^{16}$ Taken together, the above studies demonstrate that comprehensive image analysis has been used across the whole spectrum of TIC studies to leverage the maximum amount of information from tissue based biomarkers.

Image analysis has been employed extensively for the study of TICs in glioblastomas, going beyond analysis of single markerdefined populations to morphologic analysis and KI67 proliferation index in the analysis of lineage hierarchy ${ }^{17}$ and quantitative profiling of a broad range of histological markers of TIC populations with integration of genomic assessments. ${ }^{18}$ These studies highlight the power of image analysis to help understand tumors of complex biology such as GBM, while providing potentially clinical relevant information that can help bridge genomic and histologic biomarker approaches while enhancing the interpretive power of both. Taking this powerful paradigm for integrating multiple data types can be enhanced further through the application of data mining and machine 
learning techniques, which have already demonstrated the ability to improve patient selection for clinical trials in the immune oncology field. ${ }^{1}$ Learnings from this field can be applied to trials focused on TICs, while simplifying the additional complexity of layering TIC profiling of tumors with immuno profiling and genomic information to arrive at a more comprehensive understanding of treatment response, resistance, and recurrence in all tumor types. Considering the future of combination therapies, a dizzying array of potential components will be available to each physician tailoring care to their patients: TIC targeting, immunotherapy, antibody-drug conjugates, oncolytic viruses, therapeutic enzymes, traditional chemo and radiation, and many other treatment options represent potential choices for the physician and patient to navigate. While transcriptional analyses might be able to assess these in a single assay, spatial context critical to optimum patient selection ${ }^{1}$ would be lost. To effectively determine which therapeutic regimen best suits a broad range of patients, approaches combining the full spectrum of targets with tissue context must be employed. CNT based image analysis combined with data mining and machine learning techniques could offer such an approach, which could address the complexity of targeting TICs as well as other treatment modalities in the context of personalized medicine.

\section{Acknowledgements}

None.

\section{Conflict of interest}

The author declares no conflict of interest.

\section{References}

1. Althammer S, Steele K, Rebelatto M, et al. Combinatorial CD8+ and $\mathrm{PD}-\mathrm{L} 1+$ cell densities correlate with response and improved survival in non-small cell lung cancer (NSCLC) patients treated with durvalumab. $J$ Immunother Cancer. 2016;4:191.

2. Williams KE, Bundred NJ, Landberg G, et al. Focal adhesion kinase and wnt signalling regulates human ductal carcinoma in situ stem cell activity and response to radiotherapy. Stem Cells Dayt Ohio. 2014;33(2):327-341

3. Maccalli C, Parmiani G, Ferrone S. Immuno modulating and Immuno resistance Properties of Cancer-Initiating Cells: Implications for the Clinical Success of Immunotherapy. Immunol Invest. 2017;46(3):221238.

4. Malladi S, Macalinao DG, Jin X, et al. Metastatic Latency and Immune Evasion through Autocrine Inhibition of WNT. Cell. 2016;165(1):4560
5. Bernardes de Jesus B, Vera E, Schneeberger K, et al. Telomerase gene therapy in adult and old mice delays aging and increases longevity without increasing cancer. EMBO Mol Med. 2012;4(8):691-704.

6. Varela E, Muñoz-Lorente MA, Tejera AM, et al. Generation of mice with longer and better preserved telomeres in the absence of genetic manipulations. Nat Commun. 2016;7:11739.

7. Baatz M, Zimmermann J, Blackmore CG. Automated analysis and detailed quantification of biomedical images using Definiens Cognition Network Technology. Comb Chem High Throughput Screen. 2009;12(9):908-916.

8. Flores I, Canela A, Vera E, et al. The longest telomeres: a general signature of adult stem cell compartments. Genes Dev. 2008;22(5):654-667.

9. Chen HF, Yu CY, Chen MJ, et al. Characteristic expression of major histocompatibility complex and immune privilege genes in human pluripotent stem cells and their derivatives. Cell Transplant. $2015 ; 24(5): 845-864$.

10. Ichiryu N, Fairchild PJ. Immune privilege of stem cells. Methods Mol Biol Clifton NJ. 2013;1029:1-16.

11. Abraham BK, Fritz P, McClellan M, et al. Prevalence of CD44+ CD24-/low cells in breast cancer may not be associated with clinical outcome but may favor distant metastasis. Clin Cancer Res. 2005;11(3):1154-1159.

12. Sheridan C, Kishimoto H, Fuchs RK, et al. CD44+/CD24- breast cancer cells exhibit enhanced invasive properties: an early step necessary for metastasis. Breast Cancer Res. 2006;8(5):R59.

13. Ghuwalewala S, Ghatak D, Das P, et al. CD44(high)CD24(low) molecular signature determines the Cancer Stem Cell and EMT phenotype in Oral Squamous Cell Carcinoma. Stem Cell Res. 2016;16(2):405-417.

14. Lin L, Jou D, Wang Y, et al. STAT3 as a potential therapeutic target in $\mathrm{ALDH}+$ and CD44+/CD24+ stem cell-like pancreatic cancer cells. Int J Oncol. 2016;49(6):2265-2274.

15. Navas T, Pfister TD, Lawrence SM, et al. Abstract 5082: Impact of HGF knockin microenvironment on epithelial-mesenchymal transition and cancer stem cells in a non-small cell lung cancer xenograft model. Cancer Res. 2015;75:5082-5082.

16. Gedye C, Sirskyj D, Lobo NC, et al. Cancer stem cells are underestimated by standard experimental methods in clear cell renal cell carcinoma. Sci Rep. 2016;6:25220.

17. Chen R, Nishimura MC, Bumbaca SM, et al. A hierarchy of self-renewing tumor-initiating cell types in glioblastoma. Cancer Cell. 2010;17(4):362-375

18. Henriquez NV, Forshew T, Tatevossian R, et al. Comparative expression analysis reveals lineage relationships between human and murine gliomas and a dominance of glial signatures during tumour propagation in vitro. Cancer Res. 2013;73(18):5834-5844. 